\title{
Charge and Magnetic Flux Correlations in Chern-Simons Theory with Fermions
}

\author{
E.C.MARINO \\ Instituto de Física \\ Universidade Federal do Rio de Janeiro \\ Cx.P. 68528 \\ Rio de Janeiro RJ 21945-970 \\ Brasil
}

\begin{abstract}
Charge and magnetic flux bearing operators are introduced in Chern-Simons theory both in its pure form and when it is coupled to fermions. The magnetic flux creation operator turns out to be the Wilson line. The euclidean correlation functions of these operators are shown to be local and are evaluated exactly in the pure case and through an expansion in the inverse fermion mass whenever these are present. Physical states only occur in the presence of fermions and consist of composite charge-magnetic flux carrying states which are in general anyonic. The large distance behavior of the correlation functions indicates the condensation of charge and magnetic flux.
\end{abstract}

* Work supported in part by CNPq-Brazilian National Research Council. E-Mail address: marino@if.ufrj.br 


\section{1) Introduction}

Chern-Simons theory presents several peculiar properties which made it the object of intense investigation in the few past years. Besides the interest in the purely formal aspects of it there is also a vast potential of phenomenological applications in the physics of condensed matter, more specifically in the quantum Hall effect and high temperature superconductivity.

In this work we consider Chern-Simons theory, both in its pure form and when it is coupled to fermions and investigate the properties of some coherent-like states of the gauge field which carry either magnetic flux, charge or both. The idea is to construct the creation operators for such states by following a general procedure which was applied before [1, 2, 3] (see also [4, 5] for related works) in theories containing Maxwell terms in the lagrangian. As it turns out, the magnetic flux (vortex) creation operator is the Wilson line and its correlation functions are proved to be local (line independent) in Chern-Simons theories. In the absence of fermions, the quantum vortex states are unphysical, because they do not satisfy the condition imposed by the field equation on physical states. When fermions are coupled to the Chern-Simons field, we show that the only physical states are the ones created by composite charge-magnetic-flux carrying operators which are in general anyonic, except for a specific value of the $\theta$ parameter of the Chern-Simons theory. We evaluate the correlation functions of them through an expansion in the inverse fermion mass. The large distance behavior of these indicate a total condensation of charge and magnetic flux.

In Section 2 we consider pure Chern-Simons theory and introduce creation operators for vortex-like excitations examining the condition for locality of their correlation functions. The relevant commutators of these operators as well as their correlation functions are evaluated. We also introduce the $\sigma$ - operators which, in the presence of fermions, will become the creation operators of charged states.

In Section 3, we couple fermions to the theory and determine the corresponding form of the operators carrying charge and magnetic flux under the condition of locality of their correlation functions. We also evaluate the basic commutators and the explicit 
form of the correlation functions.

Perspectives of application for the work developed here are presented in Section 4. Four Appendixes are included to demonstrate useful results.

\section{2) Pure Chern-Simons Theory}

\section{1) Flux Carrying Operators}

Let us consider in this section pure Chern-Simons theory, whose lagrangian is given by

$$
\mathcal{L}=\frac{\theta}{4} \epsilon^{\mu \alpha \beta} A_{\mu} F_{\alpha \beta}
$$

where $\theta$ is an arbitrary real number. We want to obtain an operator which creates states bearing magnetic flux and whose correlation functions are local. In a previous work [1], we showed that in Maxwell-type theories such an operator could be obtained by requiring that its correlation functions were given by the vacuum functional in the presence of the external field

$$
\tilde{B}^{\mu \nu}(z ; x)=b \int_{x, L}^{\infty} d \xi_{\lambda} \epsilon^{\lambda \mu \nu} \delta^{3}(z-\xi)
$$

which would be coupled through the shift $F^{\mu \nu} \rightarrow F^{\mu \nu}+\tilde{B}^{\mu \nu}$. The resulting correlation functions were shown to be independent of the curve $L$ appearing in the definition of $\tilde{B}_{\mu \nu}$ in (2.2), being therefore local. In the same way, we are going to introduce a magnetic flux carrying operator in Chern-Simons theory by expressing its euclidean correlation function as

$$
<\mu(x) \mu^{\dagger}(y)>=Z^{-1} \int D A_{\mu} \exp \left\{-\int d^{3} z\left[-\frac{i \theta}{4} \epsilon^{\mu \alpha \beta} A_{\mu}\left(F_{\alpha \beta}+2 \tilde{B}_{\alpha \beta}(x, y)\right)\right]\right\}
$$

where $\tilde{B}(x, y)=\tilde{B}(z, x)-\tilde{B}(z, y)$ with $\tilde{B}(z, x)$ given by 2.2). Notice the factor 2 in the above expression. As we will see, this will compensate for the fact that the Chern-Simons lagrangian is not completely expressed in terms of $F_{\mu \nu}$ as in the case of Maxwell. 
From the above expression we can extract the form of the flux carrying operator, namely

$$
\begin{gathered}
\mu(x)=\exp \left\{\frac{i \theta}{2} \int d^{3} z \epsilon^{\mu \alpha \beta} A_{\mu} \tilde{B}_{\alpha \beta}\right\} \\
\mu(x)=\exp \left\{-i b \theta \int_{x, L}^{\infty} d \xi^{\mu} A_{\mu}\right\}
\end{gathered}
$$

where in the last step we already went back to Minkowski space. We see that $\mu$ is the Wilson line operator. We will show that in Chern-Simons theory it creates sates carrying magnetic flux and possesses local ( $L$-independent) correlation functions.

We can show the path independence of (2.3) by making the change of variable

$$
\begin{gathered}
A_{\mu} \rightarrow A_{\mu}-\Omega_{\mu} \\
\Omega_{\mu}=b \int_{S\left(L, L^{\prime}\right)} d^{2} \xi_{\mu} \delta^{3}(z-\xi)
\end{gathered}
$$

where $L^{\prime}$ is an arbitrary path going from $x$ (or $y$ ) to infinity, $S\left(L, L^{\prime}\right.$ ) is the surface bounded by $L$ and $L^{\prime}$ and $d^{2} \xi^{\mu}$ its surface element. Under the above change of variable, the exponent in (2.3) changes as (see Appendix A)

$$
S_{C S}\left[\tilde{B}_{\mu \nu}(L)\right] \rightarrow S_{C S}\left[\tilde{B}_{\mu \nu}\left(L^{\prime}\right)\right]
$$

and threfore the path independence of $\mu$-correlation functions is established.

Let us evaluate now the equal time commutator of $\mu$ with the magnetic flux operator

$$
\Phi=\int d^{2} x \epsilon^{i j} \partial_{i} A_{j}
$$

Using (2.4), we have

$$
\begin{aligned}
{[\mu(x), \Phi] } & =\mu(x)(-i b \theta) \int d^{2} y \int_{x, L}^{\infty} d \xi^{k} \epsilon^{i j} \partial_{i}^{(y)}\left[A_{k}(\xi), A_{j}(y)\right] \\
& =\mu(x) b \int d^{2} y \int_{x, L}^{\infty} d \xi^{i} \partial_{i}^{(y)} \delta^{2}(\xi-y)=b \mu(x)
\end{aligned}
$$

where we used the equal-time commutator $\left[A_{i}(x), A_{j}(y)\right]=\frac{i}{\theta} \epsilon^{i j} \delta^{2}(\vec{x}-\vec{y})$. This shows that indeed the $\mu$-operator introduced above carries $b$ units of magnetic flux. It is therefore a "vortex" creation operator. 


\section{2) $\quad \sigma$-Operators}

Let us intruduce now an operator called $\sigma$ which plays an interesting role, in connection with the magnetic flux carrying operator $\mu$. In Maxwell theory [6] $\sigma$ would create charged states, being the dual of $\mu$, in the sense of order-disorder duality. Also when coupling charged fermions to the Chern-Simons field (see next Section) we will see that $\sigma$ becomes a charge carrying operator. Most important of all, however, is the fact that the physical states of the theory will be created by the composite operator $\sigma \mu$.

Again, following the procedure taken for $\mu$, we will introduce local $\sigma$ correlation functions by coupling to $F_{\mu \nu}$ an external field which was used in Maxwell type theories in order to describe the analogous charged operators [6], namely

$$
\tilde{C}^{\alpha \beta}(z ; x)=\partial^{\alpha} \tilde{C}^{\beta}(z ; x)-\partial^{\beta} \tilde{C}^{\alpha}(z ; x)
$$

with

$$
\tilde{C}^{\alpha}(z ; x)=i a \int_{x, L}^{\infty} d \xi^{\alpha} \delta^{3}(z-\xi)
$$

We introduce the euclidean $\sigma$-correlation functions through

$$
<\sigma(x) \sigma^{\dagger}(y)>=Z^{-1} \int D A_{\mu} \exp \left\{-\int d^{3} z\left[-\frac{i \theta}{4} \epsilon^{\mu \alpha \beta} A_{\mu}\left(F_{\alpha \beta}+2 \tilde{C}_{\alpha \beta}(x, y)\right)\right]\right\}
$$

where $\tilde{C}(x, y)=\tilde{C}(z, x)-\tilde{C}(z, y)$ with $\tilde{C}(z, x)$ given by (2.8).

Now, from (2.9) we can derive the form of the $\sigma$-operator

$$
\begin{gathered}
\sigma(x)=\exp \left\{i a \theta \int d^{3} z \epsilon^{\mu \alpha \beta} A_{\mu} \partial_{\alpha} \tilde{C}_{\beta}\right\} \\
\sigma(x)=\exp \left\{i a \theta \int_{x, L}^{\infty} d \xi^{i} \epsilon^{i j} E_{j}\right\}
\end{gathered}
$$

where we went back to Minkowski space in the last step and chose a spatial path. $E^{i}$ is the electric field. From the second expression for $\sigma$ in (2.10) we see that it commutes with the magnetic flux operator $\Phi$.

As for the case of $\mu$, let us show the path independence of (2.9). We do this by making in (2.9) the change of variable

$$
A_{\mu} \rightarrow A_{\mu}-\tilde{\Omega}_{\mu}
$$




$$
\tilde{\Omega}_{\mu}=a \oint_{L^{\prime}-L} d \xi_{\mu} \delta^{3}(z-\xi)
$$

where $L^{\prime}$ is an arbitrary path going from $x$ (or $y$ ) to infinity. Under the above change of variable, the exponent in (2.9) changes as (see Appendix B)

$$
S_{C S}\left[\tilde{C}_{\mu \nu}(L)\right] \rightarrow S_{C S}\left[\tilde{C}_{\mu \nu}\left(L^{\prime}\right)\right]
$$

and therefore the correlation function (2.9) is path independent.

\section{3) Correlation Functions}

Let us evaluate in this subsection the correlation functions involving the operators $\sigma$ and $\mu$ introduced previously. We start with the mixed correlation function which according to (2.3) and (2.9) is given by

$$
\begin{gathered}
<\sigma\left(x_{1}\right) \mu\left(x_{2}\right) \sigma^{\dagger}\left(y_{1}\right) \mu^{\dagger}\left(y_{2}\right)>=Z^{-1} \int D A_{\mu} \exp \left\{-\int d^{3} z\left[-\frac{i \theta}{4} \epsilon^{\mu \alpha \beta} A_{\mu}\left(F_{\alpha \beta}+\right.\right.\right. \\
\left.\left.\left.2 \tilde{B}_{\alpha \beta}\left(x_{2}, y_{2}\right)+2 \tilde{C}_{\alpha \beta}\left(x_{1}, y_{1}\right)\right)\right]\right\}
\end{gathered}
$$

This quadractic functional integral can be evaluated through the use of the euclidean propagator of the Chern-Simons field,

$$
D^{\mu \nu}(x)=\frac{i}{\theta} \epsilon^{\mu \alpha \nu} \partial_{\alpha} \frac{1}{4 \pi|x|}+\text { gauge terms }
$$

giving the result

$$
<\sigma \mu \sigma^{\dagger} \mu^{\dagger}>=\exp \left\{\frac{i}{2 \theta} \int d^{3} z d^{3} z^{\prime}\left[B_{\mu}+D_{\mu}\right](z)\left[B_{\nu}+D_{\nu}\right]\left(z^{\prime}\right) \epsilon^{\mu \alpha \nu} \partial_{\alpha}\left[\frac{1}{4 \pi\left|z-z^{\prime}\right|}\right]\right\}
$$

where

$$
\begin{array}{r}
B_{\mu}=\frac{-i \theta}{2} \epsilon^{\mu \alpha \beta}\left[\tilde{B}_{\alpha \beta}\left(x_{2}\right)-\tilde{B}_{\alpha \beta}\left(y_{2}\right)\right]=-i b \theta \int_{x_{2}, L}^{y_{2}} d \xi_{\mu} \delta^{3}(z-\xi) \\
D_{\mu}=-i \theta \epsilon^{\mu \alpha \beta} \partial_{\beta}\left[\tilde{C}_{\alpha}\left(x_{1}\right)-\tilde{C}_{\alpha}\left(y_{1}\right)\right]=a \theta \int_{x_{1}, L}^{y_{1}} d \xi_{\lambda} \epsilon^{\lambda \alpha \mu} \partial_{\alpha} \delta^{3}(z-\xi)
\end{array}
$$

The exponent in (2.15) will have three terms: $B B, D D$ and $B D$. Let us first consider the $D D$ term. Inserting $D_{\mu}$, integrating over $z$ and $z^{\prime}$ and using a trivial identity for the $\epsilon$ 's we get

$$
D D=-i a^{2} \theta \int_{x_{1}, L}^{y_{1}} d \xi_{\lambda} \int_{x_{1}, L}^{y_{1}} d \eta_{\mu} \epsilon^{\lambda \alpha \mu} \partial_{\alpha} \delta^{3}(\xi-\eta)=0
$$


This is zero because $d \xi_{\lambda} \| d \eta_{\mu}$ for $\xi=\eta$. Let us turn now to the $B D$ term. Inserting (2.16) in (2.15), and following the same steps which led to (2.17) we get

$$
B D=a b \theta \int_{x_{2}, L}^{y_{2}} d \xi_{\mu} \int_{x_{1}, L^{\prime}}^{y_{1}} d \eta_{\nu}\left[\delta^{\mu \nu}(-\square)-\partial_{(\xi)}^{\mu} \partial_{(\eta)}^{\nu}\left[\frac{1}{4 \pi|\xi-\eta|}\right]\right.
$$

The expression between brackets is the Green function of $-\square$. Hence, the first term above is proportional to $\delta^{3}(\xi-\eta)$ and vanishes because we can always choose the curves $L$ and $L^{\prime}$ as nonintercepting. Evaluation of the second term is trivial and gives

$$
B D=\theta \frac{a b}{4 \pi}\left[\frac{1}{\left|y_{1}-x_{2}\right|}+\frac{1}{\left|y_{2}-x_{1}\right|}-\frac{1}{\left|y_{2}-y_{1}\right|}-\frac{1}{\left|x_{2}-x_{1}\right|}\right]
$$

Now for the $B B$ term. Using (2.2) and (2.14) we obtain

$$
B B=-\frac{i b^{2} \theta}{2} \int_{x_{2}, L}^{y_{2}} d \xi_{\mu} \int_{x_{2}, L}^{y_{2}} d \eta_{\nu} \epsilon^{\mu \alpha \nu} \partial_{\alpha}^{(\xi)}\left[\frac{1}{4 \pi|\xi-\eta|}\right]
$$

In Appendix $\mathrm{C}$ we show that this expression gives

$$
B B=-i \frac{b^{2} \theta}{4 \pi}\left[\arg \left(\vec{x}_{2}-\vec{y}_{2}\right)+\arg \left(\vec{y}_{2}-\vec{x}_{2}\right)-2 \arg (\vec{\epsilon})\right]
$$

where $\epsilon$ is a regulator which we must set to zero.

Collecting all terms contributing to (2.15), we get

$$
\begin{gathered}
<\sigma\left(x_{1}\right) \mu_{R}\left(x_{2}\right) \sigma^{\dagger}\left(y_{1}\right) \mu_{R}^{\dagger}\left(y_{2}\right)>=\exp \left\{\theta \frac{a b}{4 \pi}\left[\frac{1}{\left|y_{1}-x_{2}\right|}+\frac{1}{\left|y_{2}-x_{1}\right|}-\frac{1}{\left|y_{2}-y_{1}\right|}-\frac{1}{\left|x_{2}-x_{1}\right|}\right]\right. \\
\left.-i \frac{b^{2} \theta}{4 \pi}\left[\arg \left(\vec{x}_{2}-\vec{y}_{2}\right)+\arg \left(\vec{y}_{2}-\vec{x}_{2}\right)\right]\right\}
\end{gathered}
$$

where we defined the renormalized fields

$$
\mu_{R}=\exp \left\{i \frac{b^{2} \theta}{4 \pi} \arg (\vec{\epsilon})\right\} \mu
$$

From this point it is very easy to obtain the $\left\langle\mu \mu^{\dagger}>\right.$ and $\left\langle\sigma \sigma^{\dagger}>\right.$ correlation functions. They are going to be the exponentials of the $B B$ and $D D$ terms, respectively. So we get right away

$$
<\mu_{R}(x) \mu_{R}^{\dagger}(y)>=\exp \left\{-i \frac{b^{2} \theta}{4 \pi}[\arg (\vec{x}-\vec{y})+\arg (\vec{y}-\vec{x})]\right\}
$$


and

$$
<\sigma(x) \sigma^{\dagger}(y)>=1
$$

An important remark is now in order, concerning the states whose correlation functions we are evaluating. In pure Chern-Simons theory the field equation

$$
\theta \epsilon^{\mu \alpha \beta} \partial_{\alpha} A_{\beta}=0
$$

implies that we must impose the "Gauss' Law" condition

$$
\epsilon^{i j} \partial_{i} A_{j} \mid \text { phys }>=0
$$

or

$$
\Phi \mid \text { phys }>=0
$$

on the physical states $\mid$ phys $>$. We automatically conclude that the $\mid \mu>$ states are not physical by virtue of (2.7). We could also consider the states created by the composite operator $\sigma \mu$, whose correlation functions would be easily obtained from (2.22) but we immediately see that those would also not satisfy (2.27), being therefore unphysical as well. When we couple fermions to the Chern- Simons theory, on the contrary, we will see in the next Section that the only physical states will be presicely the ones created by $\sigma \mu$. There we will study more carefully the correlation functions and properties of these composite operators.

\section{3) Chern-Simons Theory with Fermions}

\section{1) Charge and Magnetic Flux Carrying Operators}

Let us consider now the Chern-Simons theory coupled to a Dirac fermion,

$$
\mathcal{L}=\frac{\theta}{4} \epsilon^{\mu \alpha \beta} A_{\mu} F_{\alpha \beta}+i \bar{\psi} \not \supset \psi-M \bar{\psi} \psi-e \bar{\psi} \gamma^{\mu} \psi A_{\mu}
$$

The field equation is

$$
\theta \epsilon^{\mu \alpha \beta} \partial_{\alpha} A_{\beta}=e j^{\mu}
$$


where $j^{\mu}=\bar{\psi} \gamma^{\mu} \psi$. As a consequence the physical states must satisfy now the condition

$$
\theta \epsilon^{i j} \partial_{i} A_{j} \mid \text { phys }>=e j^{0} \mid \text { phys }>
$$

or

$$
\theta \Phi \mid \text { phys }>=e Q \mid \text { phys }>
$$

where $Q=\int d^{2} x j^{0}$ is the matter charge operator.

In order to obtain the local operators $\sigma$ and $\mu$ carrying respectively charge and magnetic flux in the presence of fermions, we shall follow the same procedure adopted previously and introduce the external fields $\tilde{B}_{\mu \nu}$ and $\tilde{C}^{\alpha}$ in such a way that the correlation functions involving $\sigma$ and $\mu$ are local. Starting with the $\sigma$-correlation function, we write

$$
\begin{aligned}
<\sigma(x) \sigma^{\dagger}(y)> & =Z^{-1} \int D A_{\mu} \exp \left\{-\int d^{3} z\left[-\frac{i \theta}{4} \epsilon^{\mu \alpha \beta} A_{\mu}\left(F_{\alpha \beta}+2 \tilde{C}_{\alpha \beta}(x, y)\right)\right.\right. \\
& \left.\left.+i \bar{\psi} \not \partial \psi-M \bar{\psi} \psi+e \bar{\psi} \gamma^{\mu} \psi\left[A_{\mu}+\tilde{C}_{\mu}(x, y)\right]\right]\right\}
\end{aligned}
$$

where $\tilde{C}(x, y)=\tilde{C}(z, x)-\tilde{C}(z, y)$ with $\tilde{C}(z, x)$ given by (2.8). This expression is easily seen to be path independent because of (2.11) and (2.12).

Let us turn now to the $\mu$-operator. For this, we must couple the external field $\tilde{B}_{\mu \nu}$ by adding it to $F_{\mu \nu}$. Since the action now is not completely expressed in terms of $F_{\mu \nu}$ because of the interaction term, we are going to use the gauge invariance of the fermionic determinant (at least under "small" gauge transformations) to write

$$
\begin{aligned}
<\mu(x) \mu^{\dagger}(y)> & =Z^{-1} \int D A_{\mu} \exp \left\{-\int d^{3} z\left[-\frac{i \theta}{4} \epsilon^{\mu \alpha \beta} A_{\mu}\left(F_{\alpha \beta}+2 \tilde{B}_{\alpha \beta}(x, y)\right)\right.\right. \\
& \left.\left.+i \bar{\psi} \not \partial \psi-M \bar{\psi} \psi+e \bar{\psi} \gamma_{\mu} \psi\left[\frac{\partial_{\nu}\left[F^{\mu \nu}+\tilde{B}^{\mu \nu}\right]}{-\square}\right]\right]\right\}
\end{aligned}
$$

where $\tilde{B}(x, y)=\tilde{B}(z, x)-\tilde{B}(z, y)$ with $\tilde{B}(z, x)$ given by (2.2). Notice that the action used above differs from the one corresponding to (3.1) by a gauge transformation and are therefore equivalent.

The path independence of (3.5) follows immediately from (2.5), (2.6) and the fact that under (2.5)

$$
F^{\mu \nu} \rightarrow F^{\mu \nu}+\tilde{B}^{\mu \nu}\left[L^{\prime}\right]-\tilde{B}^{\mu \nu}[L]
$$


From (3.4) and (3.5), respectively, we can infer the form of the $\sigma$ and $\mu$ operators in the presence of fermions, namely,

$$
\sigma(x)=\exp \left\{i a \theta \int_{x, L}^{\infty} d \xi^{i} \epsilon^{i j} E_{j}+i e a \theta \int_{x, L}^{\infty} d \xi^{\mu} j_{\mu}\right\}
$$

and

$$
\mu(x)=\exp \left\{-i b \theta \int_{x, L}^{\infty} d \xi^{\mu} A_{\mu}-i e b \theta \int_{x, L}^{\infty} d \xi_{\lambda} \epsilon^{\lambda \mu \nu} \partial_{\nu} \frac{j_{\mu}}{-\square}\right\}
$$

Observe that if one imposed the field equation (3.2) on the above operators one would obtain the result that the first and second terms in the exponents of (3.7) and (3.8) are identical.

Let us investigate now the commutation rules of the $\sigma$ and $\mu$ operators obtained above. It is clear that the commutators of these operators with $\Phi$ remain the same as the ones found in Section 2. In order to evaluate the commutator of $\sigma$ with the matter charge operator $Q$, let us consider the current algebra relation

$$
\left[j^{0}(\vec{x}, t), j^{i}(\vec{y}, t)\right]=i \mathcal{M} \partial^{i} \delta^{2}(\vec{x}-\vec{y})
$$

where $\mathcal{M}$ is a functional of the spectral density of the theory and has dimension of mass. Using this, we easily get

$$
\left[j^{0}(\vec{y}, t), \sigma(\vec{x}, t)\right]=\operatorname{ea\theta } \mathcal{M} \sigma(\vec{x}, t) \delta^{2}(\vec{x}-\vec{y})
$$

or

$$
[Q, \sigma(\vec{x}, t)]=\operatorname{ea\theta } \mathcal{M} \sigma(\vec{x}, t)
$$

The choice $a^{-1}=e \theta \mathcal{M}$ would imply that the operator $\sigma$ carried one unit of electric charge.

In Appendix $\mathrm{D}$ we show that the operator $\mu$ in the presence of fermions, which is given by (3.8) commutes with the charge operator.

\section{2) The Mixed Correlation Function $<\sigma \mu \sigma^{\dagger} \mu^{\dagger}>$}

Let us evaluate now the correlation functions of the charge and magnetic flux carrying operators in the presence of fermions. Using expressions (3.4) and (3.5) we write

$$
<\sigma\left(x_{1}\right) \mu\left(x_{2}\right) \sigma^{\dagger}\left(y_{1}\right) \mu^{\dagger}\left(y_{2}\right)>=Z^{-1} \int D A_{\mu} \exp \left\{-\int d^{3} z\left[-\frac{i \theta}{4} \epsilon^{\mu \alpha \beta} A_{\mu}\left(F_{\alpha \beta}\right.\right.\right.
$$




$$
\left.\left.\left.+2 \tilde{B}_{\alpha \beta}\left(x_{2}, y_{2}\right)+2 \tilde{C}_{\alpha \beta}\left(x_{1}, y_{1}\right)\right)+i \bar{\psi} \not \partial \psi-M \bar{\psi} \psi+e \bar{\psi} \gamma^{\mu} \psi\left[A_{\mu}+\tilde{C}_{\mu}+\frac{\partial_{\nu} \tilde{B}^{\mu \nu}}{-\square}\right]\right]\right\}
$$

where we again used the gauge invariance of the fermionic determinant. Let us now integrate over the fermionic field using an expansion in inverse powers of the mass $M$. The leading contribution gives [7]

$$
\begin{gathered}
<\sigma \mu \sigma^{\dagger} \mu^{\dagger}>=Z^{-1} \int D A_{\mu} \exp \left\{-\int d^{3} z\left[-\frac{i \theta}{4} \epsilon^{\mu \alpha \beta} A_{\mu}\left(F_{\alpha \beta}+2 \tilde{B}_{\alpha \beta}+2 \tilde{C}_{\alpha \beta}\right)\right.\right. \\
\left.\left.-i \frac{e^{2}}{16 \pi} \epsilon^{\mu \alpha \beta}\left[A_{\mu}+\tilde{C}_{\mu}+\frac{\partial_{\nu} \tilde{B}^{\mu \nu}}{-\square}\right] \partial_{\alpha}\left[A_{\beta}+\tilde{C}_{\beta}+\frac{\partial_{\lambda} \tilde{B}^{\beta \lambda}}{-\square}\right]\right]\right\}
\end{gathered}
$$

The last term in the exponent in the above expression yields three new contributions, which we call respctively $\overline{B B}, \overline{B C}$ and $\overline{B A}$. The $\overline{B B}$ term is given by

$$
\overline{B B}=-\frac{i b^{2} e^{2}}{16 \pi} \int_{x_{2}, L}^{y_{2}} d \xi_{\lambda} \int_{x_{2}, L}^{y_{2}} d \eta_{\gamma}\left[\epsilon^{\gamma \lambda \rho} \square \partial_{\rho}-\epsilon^{\gamma \nu \rho} \partial_{\nu} \partial_{\rho} \partial_{\lambda}\right] \delta^{3}(\xi-\eta)\left[\frac{1}{(-\square)^{2}}\right]
$$

The second term identically vanishes. The first term is also zero because $d \xi^{\lambda} \| d \eta^{\gamma}$ for $\xi=\eta$. We therefore have $\overline{B B}=0$. Let us consider now $\overline{B C}$. Inserting (2.2) and (2.8) in (3.15) we immediately find that $\overline{B C}=B D$ which is given by (2.18) and (2.19). Finally the $\overline{B A}$ term. Using (2.2) and a trivial identity involving the Levi-Civita tensors, we find

$$
\overline{B A}=-i \frac{e^{2}}{32 \pi} \int d^{3} z \epsilon^{\mu \alpha \beta} A_{\mu}\left(2 \tilde{B}_{\alpha \beta}\right)
$$

Inserting the results for these three terms in (3.15), we find

$$
\begin{gathered}
<\sigma \mu \sigma^{\dagger} \mu^{\dagger}>=Z^{-1} \int D A_{\mu} \exp \left\{-\int d^{3} z\left[-\frac{i\left(\theta+\frac{e^{2}}{8 \pi}\right)}{4} \epsilon^{\mu \alpha \beta} A_{\mu}\left(F_{\alpha \beta}+2 \tilde{B}_{\alpha \beta}+2 \tilde{C}_{\alpha \beta}\right)\right]\right\} \\
\times \exp \{\overline{B C}\}
\end{gathered}
$$

Using the results of Section 2.3, we immediately find

$$
\begin{aligned}
& <\sigma\left(x_{1}\right) \mu_{R}\left(x_{2}\right) \sigma^{\dagger}\left(y_{1}\right) \mu_{R}^{\dagger}\left(y_{2}\right)>=\exp \left\{( \theta + \frac { e ^ { 2 } } { 4 \pi } ) \frac { a b } { 4 \pi } \left[\frac{1}{\left|y_{1}-x_{2}\right|}+\frac{1}{\left|y_{2}-x_{1}\right|}\right.\right. \\
& \left.\left.-\frac{1}{\left|y_{2}-y_{1}\right|}-\frac{1}{\left|x_{2}-x_{1}\right|}\right]-i \frac{b^{2}\left(\theta+\frac{e^{2}}{8 \pi}\right)}{4 \pi}\left[\arg \left(\vec{x}_{2}-\vec{y}_{2}\right)+\arg \left(\vec{y}_{2}-\vec{x}_{2}\right)\right]\right\}
\end{aligned}
$$


This is our final result for the mixed correlation function in Chern-Simons theory coupled to fermions in leading $\frac{1}{M}$ approximation.

We can introduce a composite operator $\varphi$ bearing both charge and magnetic flux, through

$$
\varphi(x)=\lim _{x_{1} \rightarrow x_{2}} \sigma\left(x_{1}\right) \mu_{R}\left(x_{2}\right) \exp \left\{\frac{\left(\theta+\frac{e^{2}}{4 \pi}\right)}{4 \pi\left|x_{1}-x_{2}\right|}\right\}
$$

The correlation functions of $\varphi$ can be obtained from (3.16) and (C.2), giving

$$
<\varphi(x) \varphi^{\dagger}(y)>=\exp \left\{\left(\theta+\frac{e^{2}}{4 \pi}\right) \frac{a b}{2 \pi}\left[\frac{1}{|y-x|}\right]-i \frac{b^{2}\left(\theta+\frac{e^{2}}{8 \pi}\right)}{4 \pi}[\arg (\vec{x}-\vec{y})+\arg (\vec{y}-\vec{x})]\right\}
$$

The multivaluedness of this correlation function, which is a consequence of the arg functions is a reflection of the nontrivial commutation relation of the $\varphi$ field with itself [8, 9, 2]. Each sheet of (3.18) corresponds to a certain ordering of operators on the l.h.s.. The magnitude of the jump from one sheet to another in (3.18) indicates that $\varphi$ is an anyon operator with statistics $s=\frac{b^{2}\left(\theta+\frac{e^{2}}{8 \pi}\right)}{4 \pi}$.

\section{3) Physical States. Charge and Magnetic Flux Condensa- tion}

Let us analize now the consequences of imposing the physical condition (3.3) on the charge and magnetic flux carrying states introduced above. We immediately see that the $\mid \sigma>$ and $\mid \mu>$ states are both unphysical. The anyon operator, however, does create physical states in the Chern-Simons theory with fermions, provided we choose the constants $a, b$ and $\theta$ in such a way that (3.3) is fulfilled. This happens when

$$
e^{2} a \mathcal{M}=b
$$

For the special choice $a^{-1}=e \theta \mathcal{M}$ when $\sigma$ carries one unit of charge, we see that the condition for physical states becomes $\theta b=e$.

The $\varphi$-states are usually anyonic. The only exception occurs for the special value

of $\theta=-\frac{e^{2}}{8 \pi}$, for which the last term in (3.18) vanishes and $\varphi$ is bosonic

$$
<\varphi(x) \varphi^{\dagger}(y)>=\exp \left\{\frac{e^{2} a b}{16 \pi|x-y|}\right\}
$$


It is quite interesting to study the long distance behavior of the $\varphi$ correlation function. From (3.20) we see that in the bosonic case a unique limit exists, namely

$$
<\varphi(x) \varphi^{\dagger}(y)>\stackrel{|\vec{x}-\vec{y}| \rightarrow \infty}{\longrightarrow} 1
$$

This implies the condensation of charge and magnetic flux.

In the anyonic case the correlation function is multivalued, the many branches of it corresponding to various posible orderings of the scalar operators on the left hand side [8, 9, 2]. In this case one should have the condensate of bosonic bound states of anyons. We conclude that the only physical states associated with the $\sigma$ and $\mu$ operators in Chern-Simons theory with fermions form a condensate of charge and magnetic flux.

\section{4) Final Remarks}

The formalism for the description of quantum states bearing magnetic flux and charge developed in this work in the framework of Chern-Simons theory may have some interesting applications in several models for condensed matter systems which are are based on this field theory. The condensation of charge and magnetic flux in the physical states suggests interesting phenomenological implications. The formulation of correlation functions in terms of vacuum functionals in the presence of special external fields is practical and allows the obtainment of concrete results with relative ease. We intend to pursue in the near future the exploration of the possible phenomenological consequences of the results contained in this work, in connection with condensed matter systems. Another potentially interesting application would be in the ChernSimons theory coupled to a Higgs field. 


\section{A) Appendix A}

Let us demonstrate here Eq.(2.6) and thereby establish the path independence of (2.3). Inserting (2.2) in (2.3) and integrating over $z$, we immediately see that the exponent in $(2.3)$ is in the form

$$
S_{C S}\left[\tilde{B}_{\mu \nu}\right]=S_{C S}-i b \theta \int_{x_{i}, L}^{\infty} d \xi^{\mu} A_{\mu}
$$

where $x_{i}$ is either $x$ or $y$. Making the change of variable 2.5) in the above expression, we get

$$
\Delta S_{C S}\left[\tilde{B}_{\mu \nu}\right]=i \int d^{3} z \epsilon^{\mu \alpha \beta} A_{\mu} \partial_{\alpha} \Omega_{\beta}
$$

The $\Omega$ term coming from the last term in (A.1) vanishes because it is proportional to

$$
\int_{S\left(L, L^{\prime}\right)} d^{2} \xi_{\mu} \int_{x, L}^{\infty} d \eta_{\mu} \delta^{3}(\xi-\eta)=0
$$

This is zero because $d^{2} \xi^{\mu} \perp d \eta_{\mu}$ for $\xi=\eta$. Also the $\Omega-\Omega$ term coming from the first term in (A.1) vanishes because it is proportional to

$$
\int_{S\left(L, L^{\prime}\right)} d^{2} \xi_{\mu} \int_{S\left(L, L^{\prime}\right)} d^{2} \eta_{\beta} \epsilon^{\mu \alpha \beta} \partial_{\alpha} \delta^{3}(\xi-\eta)=0
$$

This is zero because $d^{2} \xi_{\mu} \| d^{2} \eta_{\beta}$ for $\xi=\eta$.

Inserting $\Omega_{\mu}$ in (A.2) and integrating over $z$, we get

$$
\begin{gathered}
\Delta S_{C S}\left[\tilde{B}_{\mu \nu}\right]=i b \theta \int_{S\left(L, L^{\prime}\right)} d^{2} \xi_{\mu} \epsilon^{\mu \alpha \beta} \partial_{\alpha} A_{\beta} \\
=-i b \theta \oint_{L^{\prime}-L} d \xi^{\mu} A_{\mu}=S_{C S}\left[\tilde{B}\left(L^{\prime}\right)\right]-S_{C S}[\tilde{B}(L)]
\end{gathered}
$$

This demonstrates (2.6).

\section{B) Appendix B}

Let us demonstrate here Eq.(2.12) and thereby establish the path independence of (2.9). Inserting (2.8) in (2.9) and integrating over $z$, we immediately see that the exponent in (2.9) is in the form

$$
S_{C S}\left[\tilde{C}_{\mu \nu}\right]=S_{C S}+i a \theta \int_{x_{i}, L}^{\infty} d \xi^{\mu} \epsilon^{\mu \alpha \beta} \partial_{\alpha} A_{\beta}
$$


where $x_{i}$ is either $x$ or $y$. Making the change of variable (2.11) in the above expression, we get for $\Delta S_{C S}\left[\tilde{B}_{\mu \nu}\right]$ an expression identical to (A.2), with $\tilde{\Omega}$ instead of $\Omega$. Again the $\tilde{\Omega}-\tilde{\Omega}$ and $\tilde{\Omega}-\tilde{C}_{\mu \nu}$ terms vanish for the same reasons as in the previous Appendix. Inserting (2.11) in the place of $\Omega_{\mu}$ in (A.2) we get

$$
\begin{gathered}
\Delta S_{C S}\left[\tilde{C}_{\mu \nu}\right]=i a \theta \oint_{L^{\prime}-L} d \xi_{\mu} \epsilon^{\mu \alpha \beta} \partial_{\alpha} A_{\beta} \\
S_{C S}\left[\tilde{C}\left(L^{\prime}\right)\right]-S_{C S}[\tilde{C}(L)]
\end{gathered}
$$

This demonstrates (2.12).

\section{C) Appendix C}

Let us demonstrate here Eq. (2.21). We start by using the identity

$$
\epsilon^{\mu \alpha \beta} \partial_{\alpha} \Phi_{\beta}=\partial^{\mu}\left[\frac{1}{|x|}\right]
$$

or

$$
\epsilon^{\mu \nu \alpha} \partial_{\alpha}\left[\frac{1}{|x|}\right]=\partial^{\mu} \Phi^{\nu}-\partial^{\nu} \Phi^{\mu}
$$

where

$$
\Phi^{\mu}=\frac{1-\cos \theta}{r \sin \theta} \hat{\varphi}^{\mu}
$$

in spherical coordinates. Inserting (C.1) in (2.20) we get

$$
B B=\frac{i b^{2} \theta}{8 \pi}\left[\int_{x_{2}, L}^{y_{2}} d \eta_{\mu}\left[\Phi^{\mu}\left(y_{2}-\eta\right)+\Phi^{\mu}\left(\eta-y_{2}\right)\right]-\int_{x_{2}, L}^{y_{2}} d \xi_{\mu}\left[\Phi^{\mu}\left(x_{2}-\xi\right)+\Phi^{\mu}\left(\xi-x_{2}\right)\right]\right]
$$

Now, observing that

$$
\Phi^{\mu}(x-\xi)+\Phi^{\mu}(\xi-x)=2 \partial^{\mu} \arg (\vec{x}-\vec{\xi})
$$

we immediately establish (2.21) upon integration over $\xi$ and $\eta$ in (C.3). 


\section{D) Appendix D}

Let us demonstrate here that the operator $\mu$, given by (3.8) commutes with the charge operator. The first term in the exponent in (3.8) obviously commutes with the charge density $j^{0}$. The second term is

$$
J=\int d^{3} z i n t_{x, L}^{\infty} d \xi_{\lambda} \epsilon^{\lambda \mu \nu} j_{\mu}(z) \partial_{\nu}^{(\xi)} F(z-\xi)
$$

where

$$
F(x)=\frac{1}{-\square}=\frac{1}{4 \pi|x|}
$$

For convenience, we are working in euclidean space. Inserting the identity (C.1) in (D.1) and integrating in $\xi$ we get

$$
J=-\int d^{3} z j^{\mu}(z) \Phi_{\mu}(z-x)
$$

where $\Phi^{\mu}$ is given by (C.2). The piece corresponding to the last term in (C.1) vanishes because we can always choose $L$ such that $d \xi^{\mu}$ is orthogonal to $\Phi^{\mu}$. Since $j^{\mu}$ must be invariant under reversal about the $z^{3}=0$ plane as a consequence of its invariance under time-reversal in Minkowski space, only the first term of $\Phi_{\mu}$ contributes to (D.3) and we can write

$$
J=-\int d^{3} z j^{i}(z) \partial_{i} \phi(z-x)=-\int d^{3} z \partial_{0} j^{0}(z) \phi(z-x)
$$

where we integrated by parts and used the conservation of current. It is now clear that $J$ also commutes with the charge density $j^{0}$ and therefore so does the operator $\mu$. 


\section{References}

[1] E.C.Marino, Int. J. Mod. Phys. A10 (1995) 4311

[2] E.C.Marino, Phys. Rev. D38 (1988) 3194; Ann. of Phys. (NY) 224 (1993) 225;

[3] E.C.Marino, "Dual Quantization of Solitons" in Proceedings of the NATO Advanced Study Institute, Applications of Statistical and Field Theory Methods in Condensed Matter, (D.Baeriswyl, A.Bishop and J.Carmelo, Eds.) Plenum, NY, 1992.

[4] J.Fröhlich and P.A.Marchetti, Lett. Math. Phys. 16 (1988) 347; Commun. Math. Phys. 121 (1989) 177; J.Fröhlich, F.Gabiani and P.A.Marchetti, in "Proceedings, Banff Summer School in Theoretical Physics" (H.C.Lee, Ed.)

[5] G.W.Semenoff and P.Sodano, Nucl. Phys. B328 (1989) 753; M.Lüscher, Nucl. Phys. B326 (1989) 557; R.Jackiw and S.Y.Pi, Phys. Rev. D42 (1990) 3500; A.Kovner, B.Rosenstein and D.Eliezer, Nucl. Phys. B350 (1991) 325; Mod. Phys. Lett. A5 (1990) 2733; V.F.Müller, Z.Phys. C51 (1991) 665

[6] E.C.Marino, "Local Charged Operators in 2+1 D Maxwell-Type Theories", in preparation

[7] S.Deser and A.N.Redlich, Phys. Rev. Lett. 61 (1988) 1541

[8] E.C.Marino and J.A.Swieca, Nucl. Phys. B170 (1980) 175

[9] L.P.Kadanoff and H.Ceva, Phys. Rev. B3 (1971) 3918 